\title{
Eigenmode in a misaligned triangular optical cavity
}

\author{
F.Kawazoe ${ }^{1}$, R. Schilling ${ }^{2}$ and H. Lück ${ }^{1}$ \\ ${ }^{1}$ Max-Planck-Institut für Gravitationsphysik (Albert-Einstein-Institut) und Leibniz \\ Universität Hannover, Callinstr. 38, D-30167 Hannover, Germany \\ ${ }^{2}$ Max-Planck-Institut für Gravitationsphysik (Albert-Einstein-Institut), \\ Fröttmaninger Weg 18, D-85748 Garching, Germany \\ E-mail: fumiko.kawazoe@aei.mpg.de
}

\begin{abstract}
We derive relationships between various types of small misalignments on a triangular Fabry-Perot cavity and associated geometrical eigenmode changes. We focus on the changes of beam spot positions on cavity mirrors, the beam waist position, and its angle. A comparison of analytical and numerical results shows excellent agreement. The results are applicable to any triangular cavity close to an isosceles triangle, with the lengths of two sides much bigger than the other, consisting of a curved mirror and two flat mirrors yielding a waist equally separated from the two flat mirrors. This cavity shape is most commonly used in laser interferometry. The analysis presented here can easily be extended to more generic cavity shapes. The geometrical analysis not only serves as a method of checking a simulation result, but also gives an intuitive and handy tool to visualize the eigenmode of a misaligned triangular cavity.

PACS numbers: 42.79.Gn, 42.60.Da
\end{abstract}

\section{Introduction}

Fabry-Perot cavities are widely used in the field of laser interferometry, and longitudinal length shifts of a cavity mirror and the resulting change in the phase of the resonating field is well known. However in the case where suspended mirrors are used, such as in gravitational wave detectors, angular shifts play a crucial role in the detector performance; they ensure clean length control signals. Angular shifts on the cavity mirrors and resulting eigenmode changes in the circulating Gaussian beam of a plane cavity were geometrically analyzed in [1], and the results are used, together with results from simulation work, to obtain error signals to control the alignment of various cavity mirrors. Recently we designed a triangular optical cavity for the purpose of frequency stabilization for the AEI $10 \mathrm{~m}$ Prototype [2], and in the process of designing an alignment control system, a geometrical analysis for this cavity was performed. The cavity is close to an isosceles triangle, with the lengths of two sides much bigger than the other, consisting of a curved mirror and two flat mirrors yielding a waist equally separated from the two flat mirrors. However this cavity shape is most commonly used in laser interferometry, the results presented here can easily be extended to more generic cavity 
shapes. In this paper we first derive the relations of small mirror misalignments and the resulting changes in the eigenmode. By small misalignments we mean the regime where a lateral shift, and the angular deviation of the waist is smaller than the waist radius, and the divergence angle of the beam, respectively. The results are shown in terms of beam spot position changes on all the cavity mirrors, the waist position changes, and the waist angular shifts. They carry sufficient information for designing an angular control system. We then compare the results with that of two simulation tools, and show that they are in excellent agreement with each other.

\section{Types of misalignments}

Figure 1 shows the schematic of a triangular cavity when aligned. Two flat mirrors are relatively close together and are labeled as $\mathrm{M}_{\mathrm{a}}$ and $\mathrm{M}_{\mathrm{c}}$, while the curved mirror is far away, has a radius of curvature $\mathrm{R}$ and is labeled as $\mathrm{M}_{\mathrm{b}}$. The position where the beam hits the mirror $\mathrm{M}_{\mathrm{i}}$ is given by $P_{i}$, as well as the waist position by $P_{w}$, followed by the associated coordinates within the $x-y$ plane. Here, we also introduce a coordinate system attached to each of the flat mirrors $\left(y_{a}\right.$ and $\left.y_{c}\right)$ for convenience. The two equal angles of the beam at $M_{a}$ and $M_{c}$ and half the small angle at $M_{b}$ are given by $\gamma$ and $\phi$, respectively. Due to the shape of the triangle the following approximations hold and are used throughout this paper unless otherwise noted:

$$
\begin{aligned}
& \gamma \approx \pi / 2 \\
& \phi \ll 1
\end{aligned}
$$

Angular degrees of freedom in horizontal and vertical directions for the three mirrors produce six modes of misalignments.

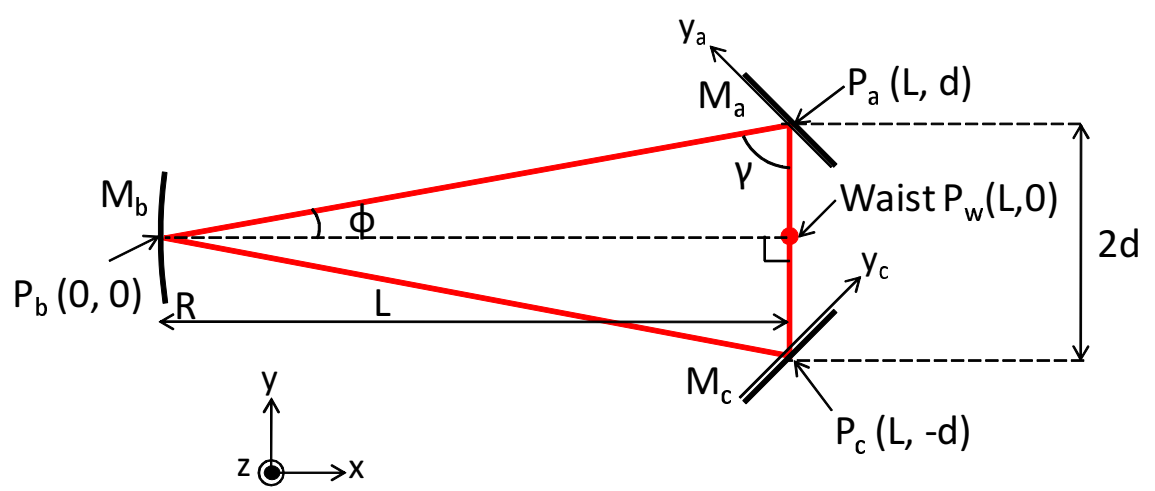

Figure 1. Schematic of an aligned triangular cavity within the $x-y$ plane. Also defined are the two coordinate axes $y_{a}$ and $y_{c}$ that are fixed on the flat mirrors $\mathrm{M}_{\mathrm{a}}$ and $\mathrm{M}_{\mathrm{c}}$, respectively. Mirror $\mathrm{M}_{\mathrm{b}}$ has a radius of curvature of $R$.

Misalignment angles of mirror $\mathrm{M}_{\mathrm{i}}$ are given by $\alpha_{i}$ and $\beta_{i}$ for horizontal (angles around the $z$-axis, sometimes also called yaw or rotation), and vertical (inclination angle with respect to the $x-y$ plane, sometimes also called pitch or tilt) directions, 
Eigenmode in a misaligned triangular optical cavity

Table 1. Summary of types of misalignments and associated section numbers.

\begin{tabular}{lll}
\hline Type & Description & Section \\
\hline$\alpha_{-}$ & Differential of the flat mirrors in horizontal & 3.1. \\
$\alpha_{b}$ & Curved mirror in horizontal & 3.2. \\
$\alpha_{+}$ & Common of the flat mirrors in horizontal & 3.3. \\
$\beta_{b}$ & Curved mirror in vertical & 4.1. \\
$\beta_{+}$ & Common of the flat mirrors in vertical & 4.2. \\
$\beta_{+}$ & Differential of the flat mirrors in vertical & 4.3. \\
\hline
\end{tabular}

respectively. A positive angle is formed by counter-clockwise rotation around the $z$-axis for horizontal misalignments, and around the $y$-axis, $y_{a}$-axis, and $y_{c}$-axis for vertical misalignment of $\mathrm{M}_{\mathrm{b}}, \mathrm{M}_{\mathrm{a}}$ and $\mathrm{M}_{\mathrm{c}}$, respectively. We take linear combinations of these two flat mirror misalignments to form common and differential modes: $\alpha_{ \pm}=\left(\alpha_{a} \pm \alpha_{c}\right) / 2$ and $\beta_{ \pm}=\left(\beta_{a} \pm \beta_{c}\right) / 2$. The changes in the waist position and the beam spot position on mirror $\mathrm{M}_{\mathrm{i}}$ are denoted by $\Delta k_{w}$ and $\Delta k_{i}$, with $k$ being the corresponding $x$ or $y$ coordinates. Ane angular change of the beam between the two flat mirrors is denoted by $\theta$ (see Fig. 3. Since we concern small misalignments, these changes are also small. Hence we use the following approximation throughout this paper: $\theta \ll 1$ and $O\left(\Delta k^{n}\right)=0$ for $n \geq 2$. All types of misalignments are summarized and the associated section numbers are listed in Table 1.

\section{Horizontal misalignments}

\subsection{Misalignment in $\alpha_{-}$}

A misalignment in $\alpha_{-}$, i.e. contrary tilts around the $z$-axis, keep the cavity symmetric to the $x$-axis and, hence, causes a symmetric change in the eigenmode. In Fig. 2, the original and the new eigenmodes are shown by the lighter (yellow) and darker colors (this color rule is applied throughout this paper), and the $x$ and $y$ coordinates of the spot positions on the mirrors are shown. Because of the symmetry it is obvious that $\Delta x_{a}$ equals $\Delta x_{c}$ and $\Delta x_{w}$, and due to the approximation given by Equ. 1, $\Delta y_{a}$ also equals $\Delta x_{a}$. The angle of incidence on the flat mirrors changes by $\frac{1}{2} \alpha_{-}$, as indicated by the dashed normal on one mirror surface. The large angle $\gamma^{\prime}$ changes by $-\alpha_{-}$, yielding a change by $\alpha_{-}$in half the small angle $\Delta \phi$. From looking at the shaded area in Fig. 2 we get:

$$
\Delta y_{c} \approx \sqrt{L^{2}+d^{2}} \sin \Delta \phi \approx \sqrt{L^{2}+d^{2}} \cdot \Delta \phi=-\sqrt{L^{2}+d^{2}} \cdot \alpha_{-}
$$

Therefore we know the following relations between the spot position changes and the misalignment angle:

$$
\Delta x_{a}=\Delta x_{c}=-\Delta y_{a}=\Delta y_{c}=\Delta x_{w}=-\sqrt{L^{2}+d^{2}} \cdot \alpha_{-}
$$

and hence the angle deviation $\theta$ at the waist is zero.

To summarize, a misalignment in $+(-) \alpha_{-}$causes a shrink (stretch) of the eigenmode along the $x$-axis, yielding the eigenmode to keep its isosceles shape, but change its shape in a way that it becomes more "fat" ("thin"). As a result, the waist position shifts in $x$-direction by an amount that is approximately proportional to the distance between the curved mirror and the two flat mirrors. 


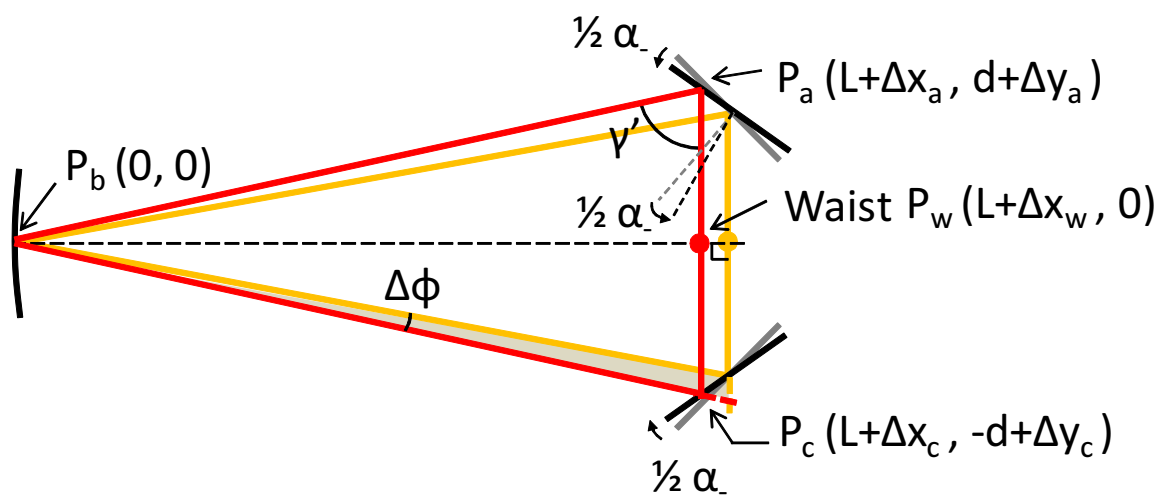

Figure 2. Cavity eigenmodes of the aligned (lighter colored triangle) and the misaligned by $\alpha_{-}$(darker colored triangle) cases. The contrary tilts around the $z$ axis cause a symmetric change in the eigenmode.

\subsection{Misalignment in $\alpha_{b}$}

Figure 3 shows a hypothetic misaligned cavity caused by $\alpha_{b}$, i.e. a rotation of $\mathrm{M}_{\mathrm{b}}$ around the vertical axis. In this case, there is no obvious symmetry axis. One can expect changes in the positions of the beam spots on the mirrors and of the waist, as well as an angle deviation at the waist. We introduce a pivot, where the non-congruent side of the aligned and the misaligned eigenmodes cross, indicated by the thick circle. We start with an arbitrary location of the pivot, and will shortly show that it coincides with the bisecting point of the non-congruent side.

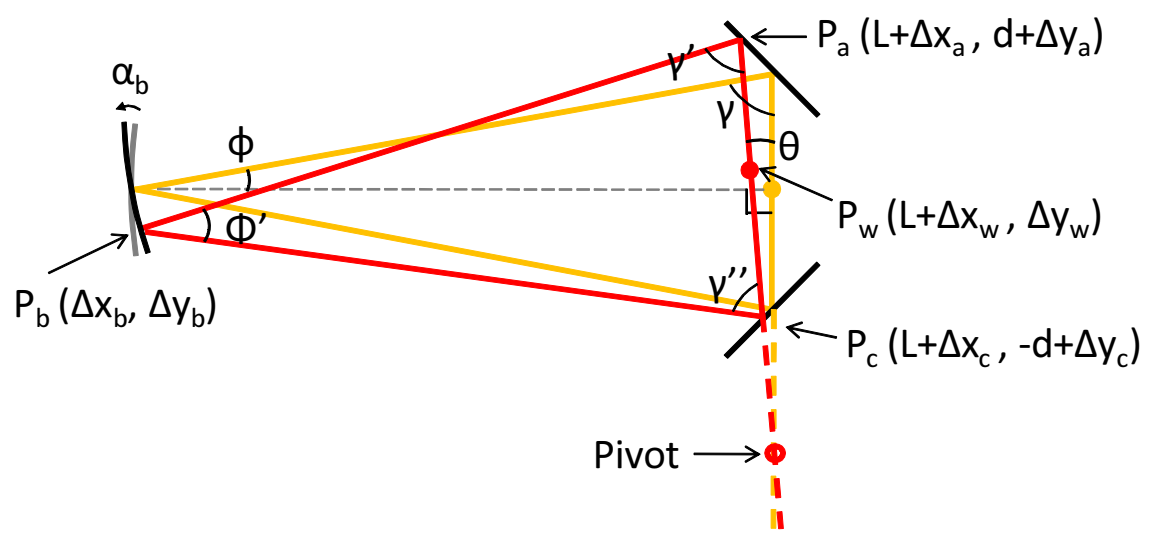

Figure 3. Cavity eigenmodes of the aligned and the misaligned cases by $\alpha_{b}$. We start with a general, and hence hypothetical case where the pivot and the bisecting point of the non-congruent do not match, and later show they coincide. The changes of the two larger angles $(\gamma)$ are of the same size but with opposite sign, hence the small angle $\phi$ stays unchanged.

The angle of incidence on the flat mirrors changes by the same amount $\theta$, but with opposite sign, resulting in the following changes of the large angles: $\gamma^{\prime}=\gamma+2 \theta$ and $\gamma^{\prime \prime}=\gamma-2 \theta$. Hence the small angle stays unchanged: $\phi^{\prime}=2 \phi$.

Looking at the flat mirrors, as shown in Fig. 4, and applying the approximation given by 
Equ. 1, one sees that $\Delta x_{a}=-\Delta y_{a}$ and $\Delta x_{c}=\Delta y_{c}$. The following set of equations describe the shift of the spot positions:

$$
\begin{aligned}
& \Delta x_{a}=l_{a} \sin \theta \\
& \begin{aligned}
\Delta x_{c} & =l_{c} \sin \theta \\
2 d & =l_{a} \cos \theta-l_{c} \cos \theta-\left|\Delta y_{a}\right|-\left|\Delta y_{c}\right| \\
& =\left(l_{a}-l_{c}\right) \cos \theta-\left(\left|\Delta x_{a}\right|+\left|\Delta x_{c}\right|\right) \\
& \approx l_{a}-l_{c}-\left(\left|l_{a}\right|+\left|l_{c}\right|\right) \theta
\end{aligned}
\end{aligned}
$$

where $l_{a}$ and $l_{c}$ are the distances from the pivot to $P_{a}$ and $P_{c}$ along the beam, respectively.

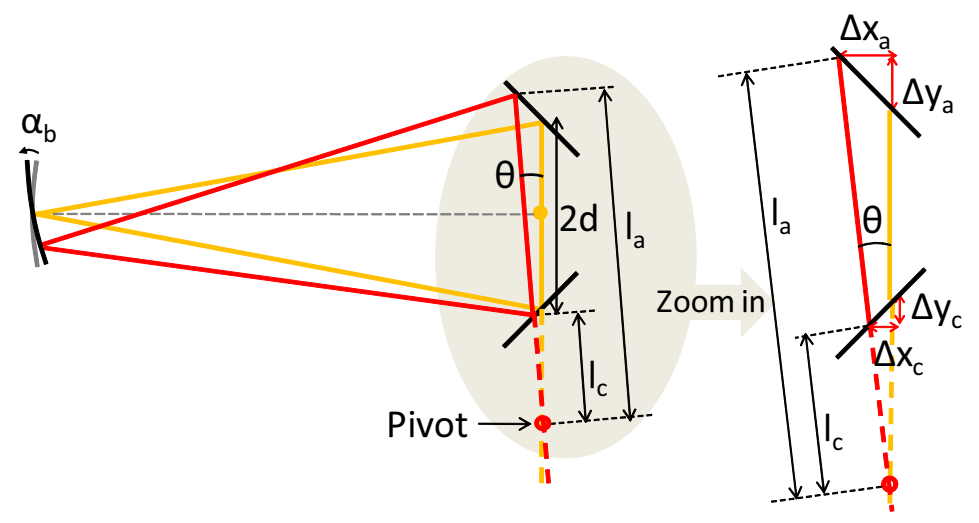

Figure 4. Closer view of the two flat mirrors and the pivot. It still shows the hypothetical eigenmode where the pivot and the bisecting point do not match.

The left hand side of Equation 7 is constant, hence the right hand side must be independent of $\theta$, yielding the following relations:

$$
\begin{aligned}
& l_{a}=-l_{c} \\
& \left|l_{a}\right|=\left|l_{c}\right|=d / \cos \theta \equiv l \\
& \Delta x_{a}=-\Delta x_{c}=-\Delta y_{a}=-\Delta y_{c}=l \sin \theta=d \tan \theta \approx d \theta
\end{aligned}
$$

This automatically means that the pivot $\left(P_{p}\right)$ bisects the non-congruent side, as shown in Fig. 5, where the changes in the location of the pivot is denoted by $\Delta x_{p}$ and $\Delta y_{p}$. It also shows the details around the flat mirrors, from which the pivot location with respect to the original waist is given by

$$
\begin{aligned}
& \Delta x_{p}=O\left(\theta^{2}\right)=0 \\
& \Delta y_{p}=d-l \cos \theta+\Delta y_{a}=-d \theta
\end{aligned}
$$

Connecting the beam spot on the curved mirror $\left(P_{b}\right)$ and the bisector point (the pivot, or $P_{p}$ ), one can see that it bisects the beam angle at $\mathrm{M}_{\mathrm{b}}$ into $\phi$, as shown in Fig. 6. This means that the line passes through the center of curvature, $\left(P_{\mathrm{COC}}\right)$, whose coordinate along the $y$-axis is given by

$$
\Delta y_{\mathrm{COC}}=R \cdot \alpha_{b}
$$

Focusing on the shaded triangles shown in Fig. 6, one can see that $\theta^{\prime}=\theta$, and, comparing the two triangles, one can also see that $\theta^{\prime \prime}=\theta^{\prime}=\theta$. The radius vectors of the aligned and 


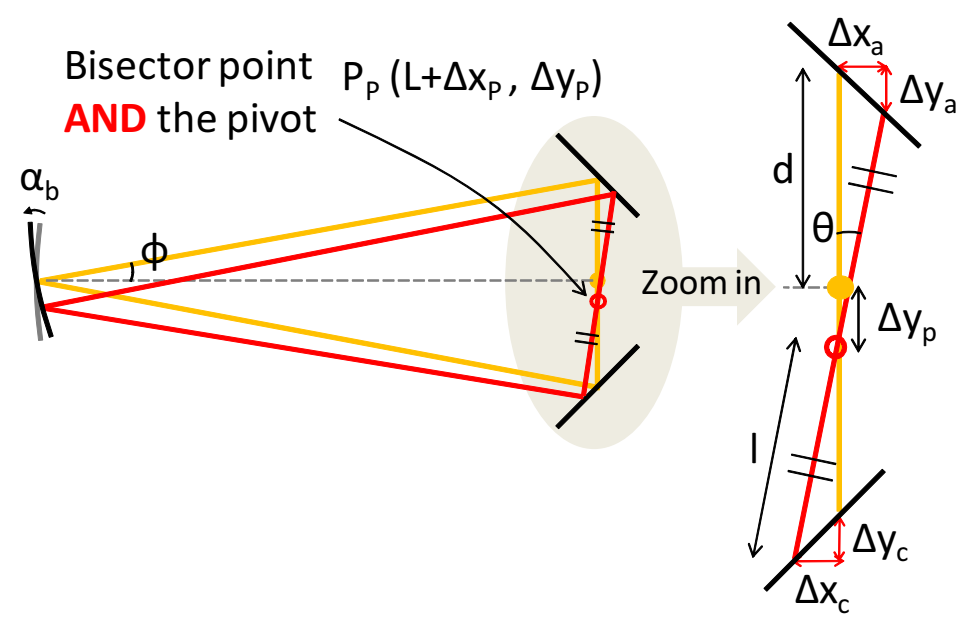

Figure 5. Closer view of the two flat mirrors. Here, the pivot and the bisecting point overlap, and the $y$ coordinate of the pivot is denoted by $\Delta y_{p}$.

misaligned mirror, indicated by the dotted lines in Fig. 6, cross at point $P_{r}$. By focusing on the triangle consisting of the original waist $\left(P_{w}\right)$, the pivot, $\left(P_{p}\right)$, and $P_{r}$, as shown in the lower triangle in Fig. 6, one can see that the $x$ coordinate of the point $P_{r}$ is given by

$$
\Delta x_{r}=d \theta / \tan \theta \approx d
$$

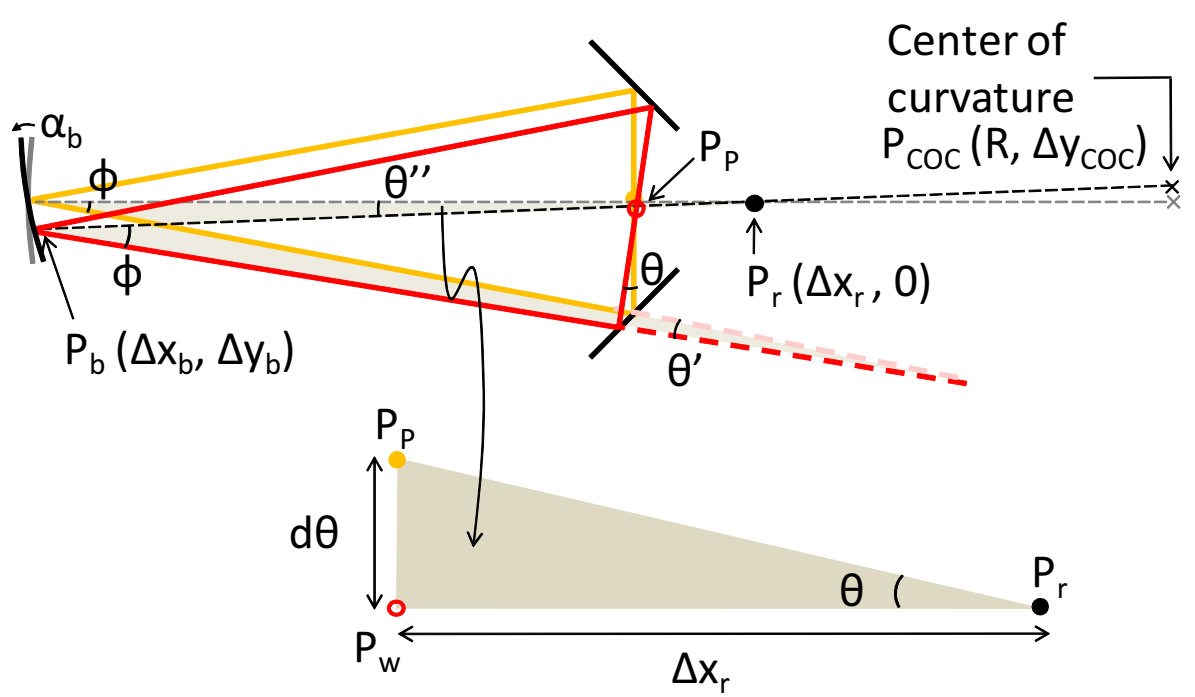

Figure 6. Radius vectors of the aligned and the misaligned cases. They cross at the point $P_{r}$, from which the angle deviation and the pivot location are calculated.

Figure 7 lists all the length information that is needed to calculate the angle $\theta$ and the spot position on $\mathrm{M}_{\mathrm{b}}$. These are given by the following set of equations:

$$
\begin{aligned}
& \theta \approx \tan \theta=R \alpha_{b} /(R-L-d) \\
& \Delta x_{b}=O\left(\Delta y_{b}^{2}\right)=0 \\
& \Delta y_{b}=-(L+d) \tan \theta \approx-(L+d) \theta=-R \alpha_{b} \cdot(L+d) /(R-L-d)
\end{aligned}
$$




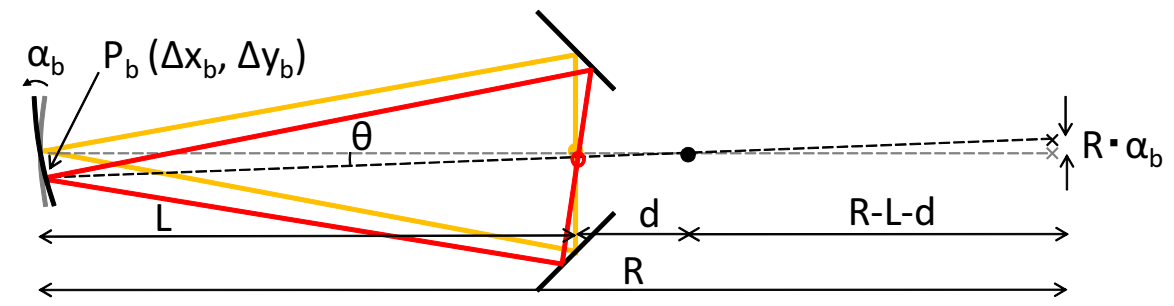

Figure 7. Length information needed to calculate $\theta$ and the spot position change on $\mathrm{M}_{\mathrm{b}}$.

Having calculated the new spot positions on the mirrors, we now calculate where the new waist is. In order for the wavefront curvature of the beam to match the radius of curvature of the curved mirror $M_{b}$, the path lengths from the waist to the mirror $M_{b}$ via $M_{a}$ and via $M_{c}$ should be the same, i.e. in Fig. 8 it should be $S_{a}+D_{a}=S_{c}+D_{c}=S+d$. By calculating the distances $S_{a}$ and $S_{b}$ in the following equations, we also will obtain the distances $D_{a}$ and $D_{b}$ :

$$
\begin{array}{rlrl}
S_{a} & =\left\{(L+d \theta)^{2}+(d+L \theta)^{2}\right\}^{1 / 2} & \\
& \approx \sqrt{L^{2}+d^{2}}\left(1+\frac{4 L d \theta}{L^{2}+d^{2}}\right)^{1 / 2} & \theta^{2}=0 \\
& \approx \sqrt{L^{2}+d^{2}}\left(1+\frac{2 L d \theta}{L^{2}+d^{2}}\right) & & \frac{2 L d \theta}{L^{2}+d^{2}} \ll 1 \\
& =S+2 d \theta & & d^{2} / L^{2}=0 \\
D_{a} & =S+d-S_{a}=d-2 d \theta &
\end{array}
$$

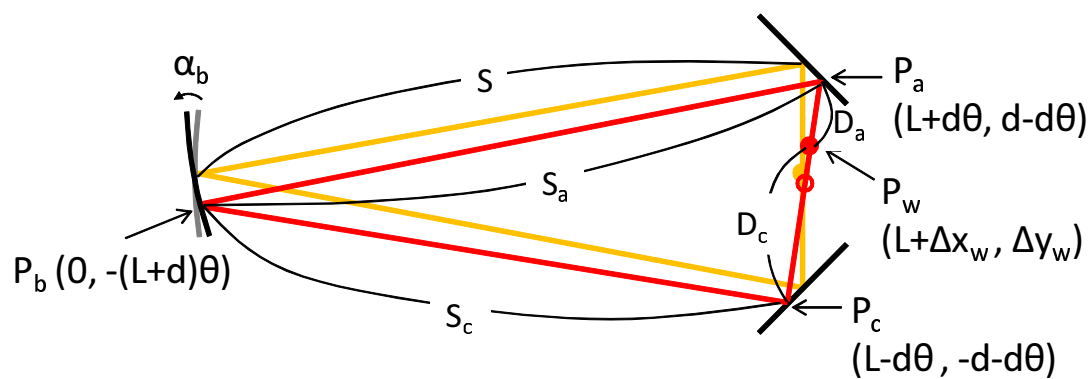

Figure 8. Locations of the new spot positions on the mirrors. By using them the new waist location is calculated.

In a similar way

$$
\begin{aligned}
& S_{c}=\left\{(L-d \theta)^{2}+(-d+L \theta)^{2}\right\}^{1 / 2}=S+2 d \theta \\
& D_{c}=S+d-S_{b}=d+2 d \theta
\end{aligned}
$$

Hence, the new waist location is given by the following:

$$
\begin{aligned}
& \Delta x_{w}=O\left(\theta^{2}\right)=0 \\
& \Delta y_{w}=\left(d-d \theta-D_{a}\right) \cos \theta \approx d \theta=d R \alpha_{b} /(R-L-d)
\end{aligned}
$$

To summarize, a misalignment in $+(-) \alpha_{b}$ causes a clockwise (counter-clockwise) rotation of the non-congruent side around the bisecting point, yielding the long sides to rotate 
synchronously. As a result all the beam spot positions change by the amounts given by the radial distances with the bisecting point being the origin of the system of radial coordinates.

\subsection{Misalignment in $\alpha_{+}$}

In the case of $\alpha_{+} \neq 0$, there is no obvious symmetry line, thus we will start from a general case. Changes on the spot positions, the beam angle at the waist, and the two larger angles are defined as shown in Fig. 9. Figure 10 focuses on the beam angle change on mirror $\mathrm{M}_{\mathrm{a}}$.

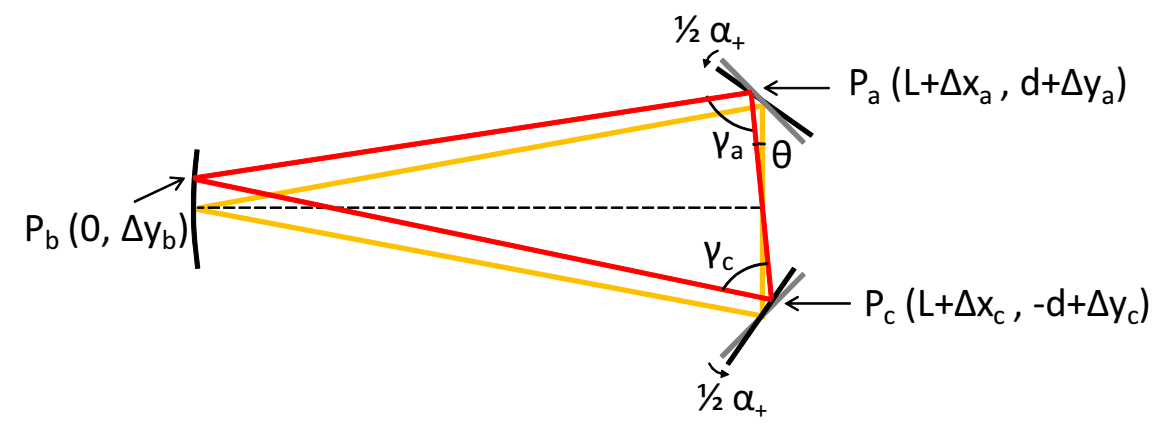

Figure 9. Cavity eigenmodes of the aligned and the misaligned $\left(\alpha_{+}\right)$cases.

Drawing helping lines such as the one that is parallel to the aligned beam (indicated by the light colored thick dotted line), as well as lines that are normal to both the aligned and the misaligned mirror surfaces (indicated by the light thin, and dark thin dotted lines, respectively) one can see that half of $\gamma_{a}$ is given by $\gamma_{a} / 2=\gamma / 2+\theta-\alpha_{+} / 2$. Hence,

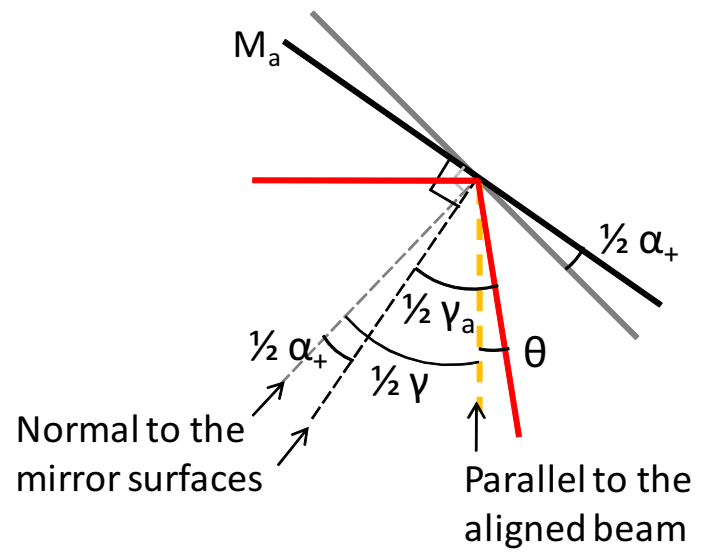

Figure 10. Closer view on the change in one of the larger angles, $\gamma_{a}$.

$$
\gamma_{a}=\gamma+\left(2 \theta-\alpha_{+}\right)
$$

In a similar manner, $\gamma_{c}$ is given by

$$
\gamma_{c}=\gamma-\left(2 \theta-\alpha_{+}\right)
$$

This means that the sum of the two angles stays unchanged, yielding no change in the small angle $\phi$. Then the line that connects $P_{b}$ with the center of curvature of $\mathrm{M}_{\mathrm{b}}$ (from here on this is called the radius), should bisect the short side, due to the fact that $d \ll L$. The bisecting 


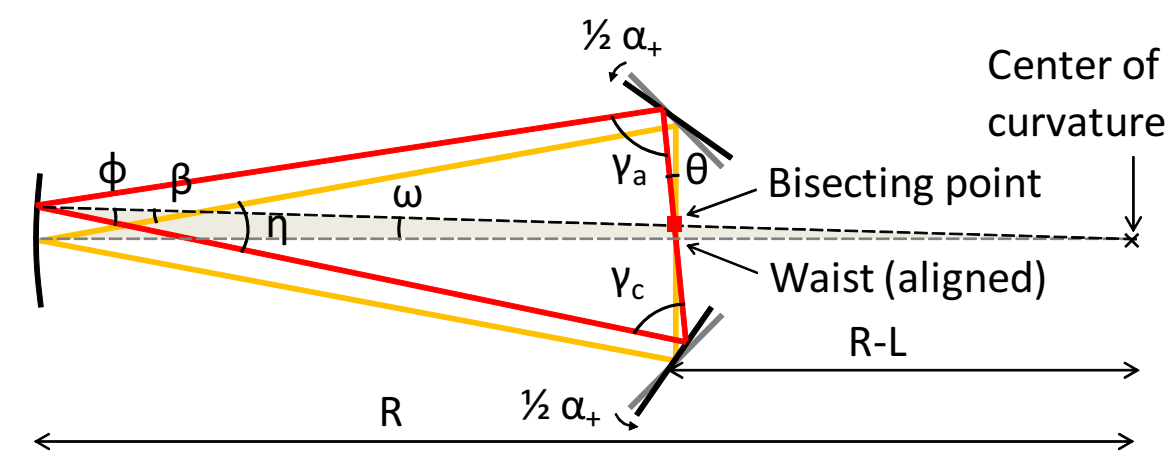

Figure 11. Ancillary angles: $\beta, \eta$ and $\omega$, which are use to calculate $\theta$.

point is indicated by the square point in Fig. 11. Here, we introduce some ancillary angles $\beta$ and $\eta$, together with $\omega$, which is the angle of the radius with respect to the aligned case. Focusing on the shaded area, one can see that the ancillary angles are given by

$$
\begin{aligned}
& \beta=\phi+\omega \\
& \eta=\phi+\beta=2 \phi+\omega
\end{aligned}
$$

$\eta$ can be expressed using $\gamma$ if one focuses on the shaded triangle shown in Fig. 12, introducing a new ancillary angle $\gamma_{a}^{\prime}=\gamma+\theta$, and it is given by

$$
\eta=\pi-\left(\gamma_{a}^{\prime}+\gamma_{c}\right)=\pi-\left\{(\gamma+\theta)+\gamma-\left(2 \theta-\alpha_{+}\right)\right\}=\pi-2 \gamma+\theta-\alpha_{+}
$$

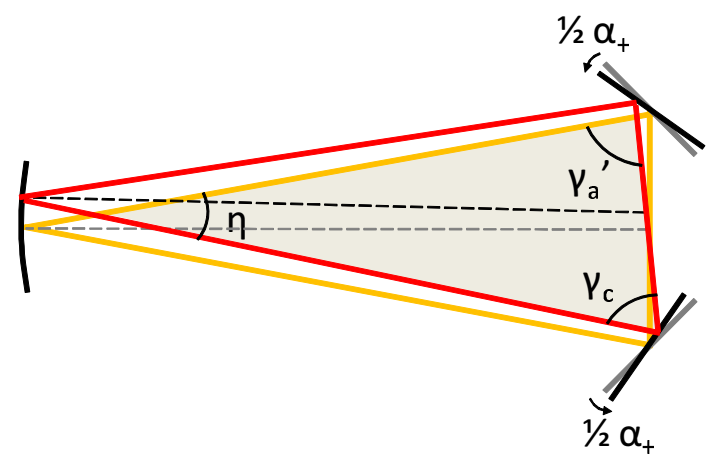

Figure 12. Yet another ancillary angle $\gamma_{a}^{\prime}$ to calculate $\eta$.

By comparing Equations 28 and 29 the angle $\omega$ is given by the following equations:

$$
\begin{aligned}
& \pi-2 \gamma+\theta-\alpha_{+}=2 \phi+\omega \\
& \omega=\pi-(2 \gamma+2 \phi)+\theta-\alpha_{+}=\theta-\alpha_{+}
\end{aligned}
$$

In order to gain additional information to finally calculate $\theta$, we focus on some lengths as shown in Fig. 13. The pivot $\left(P_{p}\right)$ is indicated by the thick circle and changes in its location are denoted by $\Delta x_{p}$ and $\Delta y_{p}$, and the two lengths from the pivot to the two beam spots by $l_{a}$ and $l_{b}$. Changes in the coordinates of the beam spot position on $\mathrm{M}_{\mathrm{a}}$ are given by the following equations:

$$
\Delta x_{a}=-l_{a} \sin \theta \approx-l_{a} \theta
$$




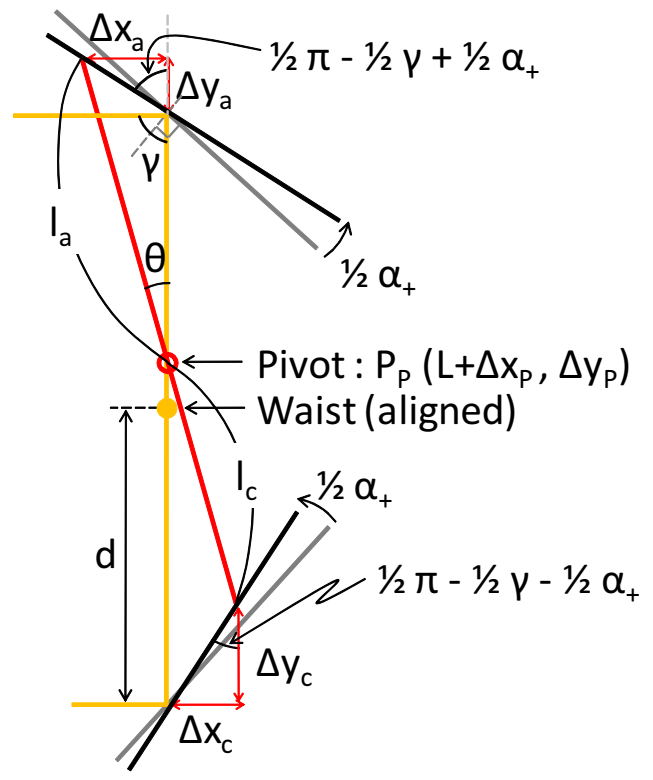

Figure 13. Length relations around the flat mirrors. From this the lengths $l_{a}$ and $l_{c}$ from the pivot to the beam spots on the two mirrors are calculated.)

$$
\Delta y_{a}=\frac{-\Delta x_{a}}{\tan \left(\pi / 2-\gamma / 2+\alpha_{+} / 2\right)} \approx \frac{1-\alpha_{+} / 2}{1+\alpha_{+} / 2} l_{a} \theta \approx\left(1-\alpha_{+}\right) l_{a} \theta
$$

and $\Delta x_{c}$ and $\Delta y_{c}$ by:

$$
\begin{aligned}
& \Delta x_{c}=l_{c} \sin \theta \approx l_{c} \theta \\
& \Delta y_{c}=\frac{\Delta x_{c}}{\tan \left(\pi / 2-\gamma / 2-\alpha_{+} / 2\right)} \approx \frac{1+\alpha_{+} / 2}{1-\alpha_{+} / 2} l_{c} \theta \approx\left(1+\alpha_{+}\right) l_{c} \theta
\end{aligned}
$$

The length of the non-congruent side is then expressed by the following:

$$
2 d=l_{a} \cos \theta+\Delta y_{a}+l_{c} \cos \theta+\Delta y_{c} \approx l_{a}+l_{c}-\left\{l_{a}-l_{c}-\left(l_{a}+l_{c}\right) \alpha_{+}\right\} \theta
$$

Since the left-hand side of Equation 36 does not depend on the misalignment angle $\theta$, the angle dependent term of the right-hand side should be zero, hence,

$$
l_{a}-l_{c}-\left(l_{a}+l_{c}\right) \alpha_{+}=0
$$

From Equations 36 and 37 the following relations can be obtained:

$$
\begin{aligned}
& l_{a}=d\left(1+\alpha_{+}\right) \\
& l_{c}=d\left(1-\alpha_{+}\right)
\end{aligned}
$$

With this knowledge we can calculate the location of the pivot in the following way:

$$
\begin{aligned}
& \Delta x_{p}=O\left(\theta^{2}\right)=0 \\
& \Delta y_{p}=l_{c} \cos \theta+\Delta y_{c}-d \approx d\left(\theta-\alpha_{+}\right)
\end{aligned}
$$

The location of the bisecting point, as shown in Fig. 14, can be calculated in a similar way, and the coordinates are given by

$$
\begin{aligned}
& \Delta x_{B}=O\left(\theta^{2}\right)=0 \\
& \Delta y_{B}=d \cos \theta+\Delta y_{c}-d \approx\left(1+\alpha_{+}\right)\left(1-\alpha_{+}\right) d \theta \approx d \theta
\end{aligned}
$$




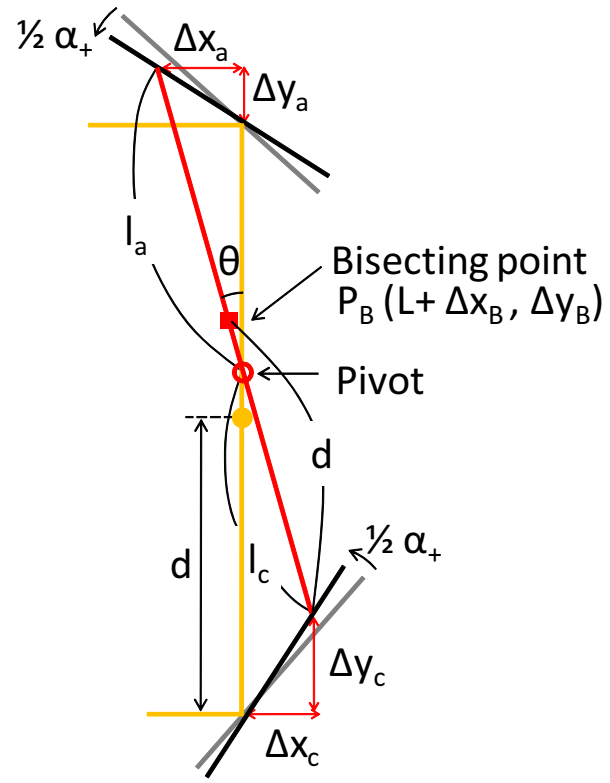

Figure 14. Length relations around the flat mirrors, including the pivot location. From this the spot position changes on the flat mirrors are calculated.

Then, focusing on the triangle that consists of the center of curvature, the waist (in the aligned case), and the bisecting point (indicated by the right part of the shaded area in Fig. 11), one can obtain another relation for $\omega$ and $\theta$ which is given by

$$
\omega \approx \tan \omega=d \theta /(R-L)
$$

From Equations 31 and 44 one can finally obtain the relation between $\theta$ and $\alpha_{+}$:

$$
\theta=\frac{R-L}{R-L-d} \cdot \alpha_{+}
$$

Using $\theta$, the spot positions on the three mirrors (see Equations 32, 33, 34, and 35) can further be calculated. This yields the following equations:

$$
\begin{aligned}
& \Delta x_{a}=-d\left(1+\alpha_{+}\right) \theta \approx-\frac{d(R-L)}{R-L-d} \cdot \alpha_{+} \\
& \Delta y_{a}=\left(1-\alpha_{+}\right) \Delta x_{a} \approx-\frac{d(R-L)}{R-L-d} \cdot \alpha_{+}
\end{aligned}
$$

And in similar ways,

$$
\begin{aligned}
& \Delta x_{c}=\frac{d(R-L)}{R-L-d} \cdot \alpha_{+} \\
& \Delta y_{c}=\frac{d(R-L)}{R-L-d} \cdot \alpha_{+}
\end{aligned}
$$

and

$$
\begin{aligned}
& \Delta x_{b}=O\left(\Delta y_{b}^{2}\right)=0 \\
& \Delta y_{b}=R \cdot \omega=R \cdot\left(\theta-\alpha_{+}\right)=\frac{d R}{R-L-d} \cdot \alpha_{+}
\end{aligned}
$$

Then the waist location can be calculated in the same way as shown in equations 19 to 24 , and the following can be shown:

$$
S_{a}=\left\{\left(L+\Delta x_{a}\right)^{2}+\left(d+\Delta y_{a}-\Delta y_{b}\right)^{2}\right\}^{1 / 2} \approx S-\left(d \theta+\Delta y_{p}\right)
$$




$$
D_{a}=d+d \theta+\Delta y_{p}
$$

In a similar way we obtain

$$
\begin{aligned}
& S_{b}=S+\left(d \theta+\Delta y_{p}\right) \\
& D_{b}=d-d \theta+\Delta y_{p}
\end{aligned}
$$

Therefore the new waist location is given by

$$
\begin{aligned}
& \Delta x_{w}=O\left(\theta^{2}\right)=0 \\
& \Delta y_{w}=\left(d+\Delta y_{a}-D_{a}\right) \cos \theta \approx-\Delta y_{p}=-\frac{d^{2}}{R-L-d} \cdot \alpha_{+}
\end{aligned}
$$

To summarize, a misalignment in $+(-) \alpha_{+}$causes a counter-clockwise (clockwise) rotation of the non-congruent side around a point that does not coincide with the bisecting point. This yields a clockwise (counter-clockwise) rotation $\omega$ (which is very small compared to the misalignment angle $\alpha_{+}$) of the geometrical axis of a corner reflector consisting of the two flat mirrors. As a result, the eigenmode changes in a "non uniform" way, with each spot position change being smaller than the misalignment case of $\alpha_{b}$.

\section{Vertical misalignments}

When considering vertical misalignments, it is necessary to view the cavity as a $3 \mathrm{D}$ body, as shown in Fig. 15. Notations of all the properties are the same as that shown in Fig. 1.

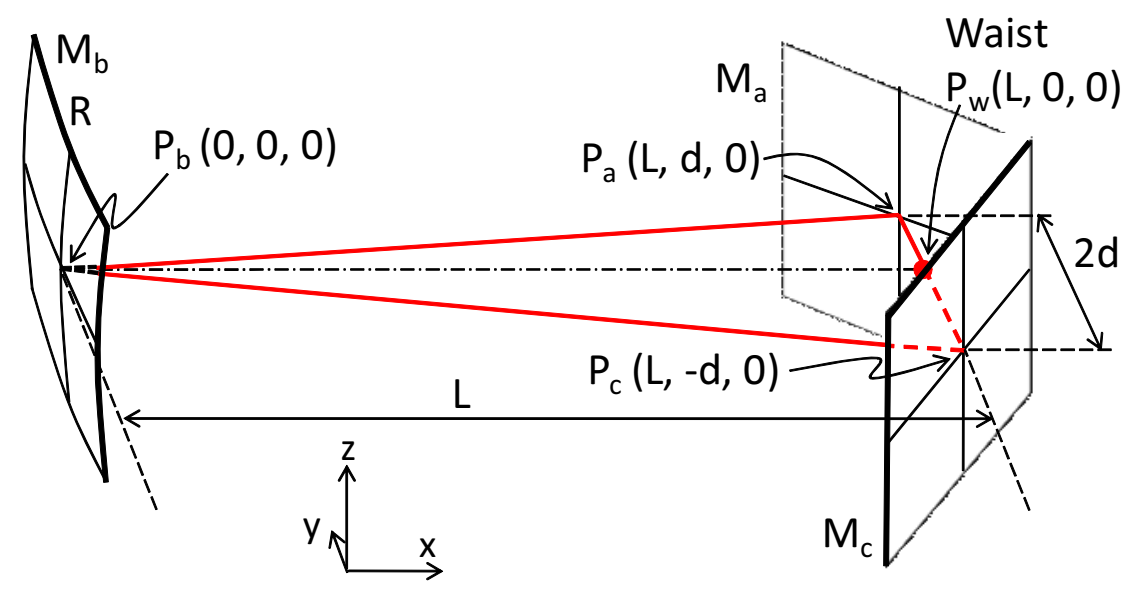

Figure 15. 3D view of a triangular cavity. $M_{a}$ and $M_{c}$ are the flat mirrors, and $M_{b}$ has a radius of curvature of $R$. The positions where the beam hits the mirror $\mathrm{M}_{\mathrm{i}}$ are denoted by $P_{i}$.

\subsection{Misalignment in $\beta_{b}$}

A misalignment around the $y$-axis by $\beta_{b}$, as shown in Fig. 16, does not affect the mirror alignment in $y$-direction, hence there is no change in eigenmode in that direction. Then it is possible to project the cavity onto the $x-z$ plane for simplicity, as shown in Fig. 17, and treat it as a plane cavity. The eigenmode of the cavity is defined by the line that is orthogonal to the flat mirrors and passes through the center of curvature, as described in [1]. It is obvious 


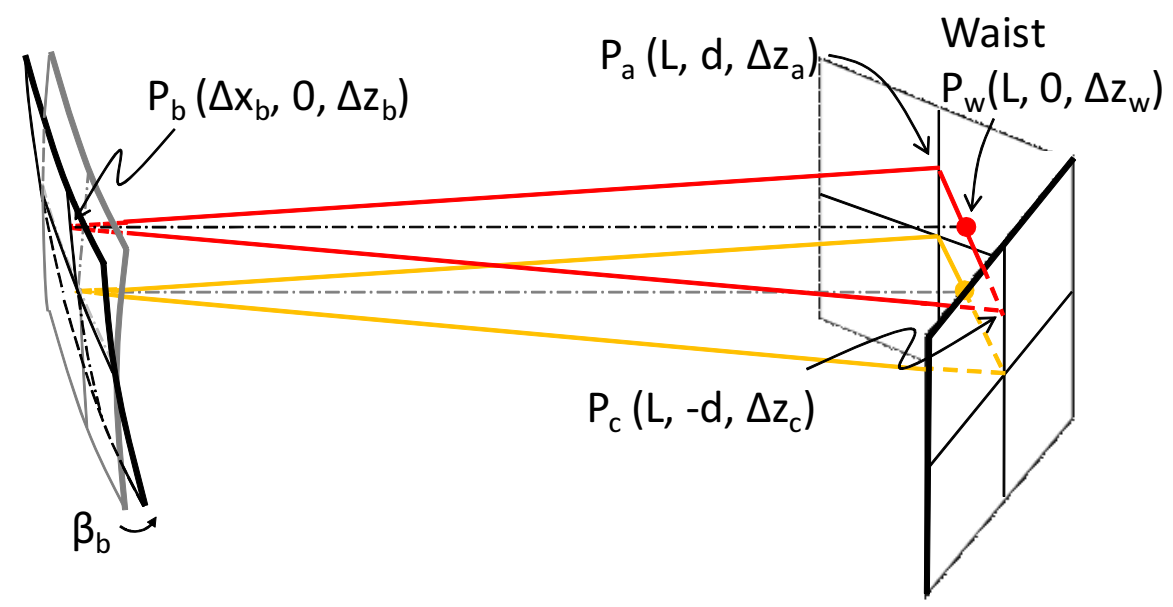

Figure 16. Cavity eigenmodes of the aligned (lighter colored triangle) and the misaligned by $\beta_{b}$ (darker colored triangle) cases. This type of misalignment does not affect the mirror alignment in $y$-direction, hence the eigenmode only changes along the $z$-axis.

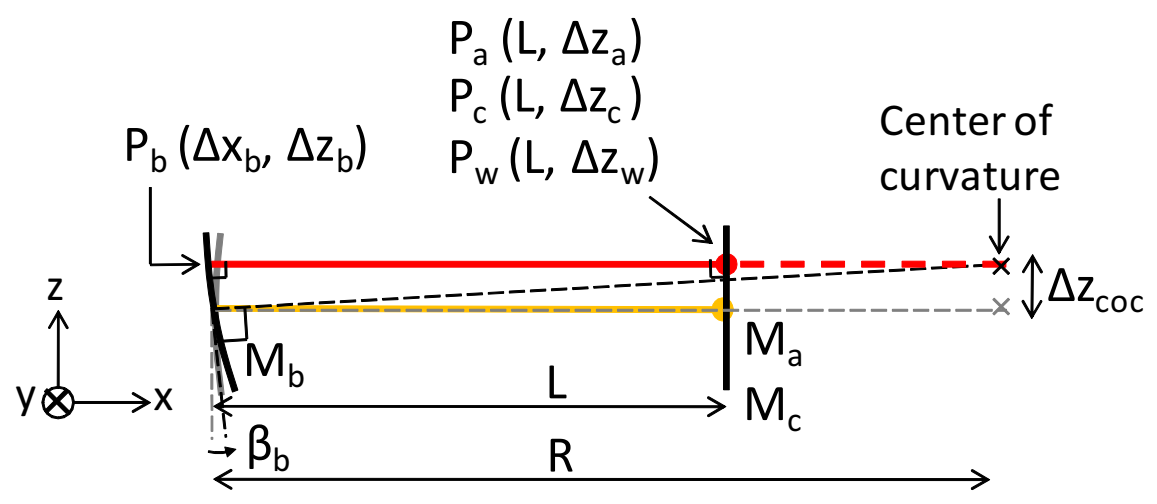

Figure 17. Projection of the triangular cavity onto the $x-z$ plane. It allows one to view the cavity as a plane cavity. The eigenmode is defined by the line that is orthogonal to the flat mirror and passes through the center of curvature.

that the eigenmode is also orthogonal to the curved mirror, yielding the shifts in $z$-direction of all of the spot positions to have the same size. The normal vector on the mirror $\mathrm{M}_{\mathrm{b}}$ is tilted by $\beta_{b}$, hence the center of curvature, whose $z$ coordinate is denoted by $z_{\text {coc }}$, shifts by $\Delta z_{\text {coc }}=\beta_{b} \cdot R$. Therefore we have the following relations:

$$
\begin{aligned}
& \Delta x_{a}=O\left(\beta_{b}^{2}\right)=0 \\
& \Delta z_{a}=\Delta z_{b}=\Delta z_{c}=\Delta z_{w}=\Delta z_{\text {coc }}=\beta_{b} \cdot R
\end{aligned}
$$

To summarize, a misalignment in $+(-) \beta_{b}$ causes an upward (downward) shift of the center of curvature along the $z$-axis, yielding a synchronous shift of the plane of the eigenmode by an amount proportional to the radius of curvature of the curved mirror. 


\subsection{Misalignment in $\beta_{+}$}

Similar to $\beta_{b}, \beta_{+}$has no effects in $y$-direction. However, since the $y_{a}$-axis and the $y_{c}$-axis are rotated by $\pm\left(\frac{\pi}{2}-\frac{\gamma}{2}\right) \approx \pm \frac{\pi}{4}$ around the $y$-axis, respectively, the projection of a misalignment by $\beta_{+} / 2$ around the two axes becomes $\frac{1}{2} \beta_{+} / \sqrt{2}$. Section 4.10 , (page 100-102) of reference [3], gives a detailed explanation of this effect by using a vector algebra and we will not describe it in this paper. For convenience we introduce an effective misalignment angle $\beta_{\text {eff }}=\beta_{+} / \sqrt{2}$.

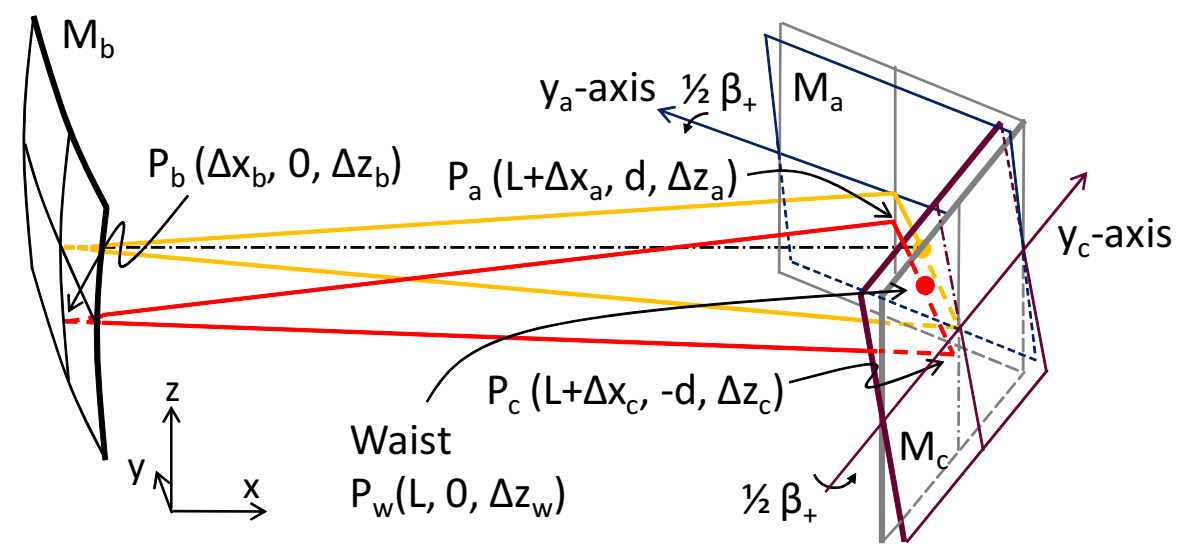

Figure 18. Cavity eigenmodes of the aligned and the misaligned $\left(\beta_{+}\right)$cases. This type of misalignment does not affect the mirror alignment in $y$-direction, hence the eigenmode only changes along the $z$-axis.

The projection of the flat mirrors are rotated by $\beta_{\text {eff }} / 2$ around the $y$-axis and the effect is doubled because of the two reflections, hence, seen as a plane cavity, the misalignment angle is given by $\beta_{\text {eff }}$, as shown in Fig. 19. The eigenmode of this cavity is defined by the line

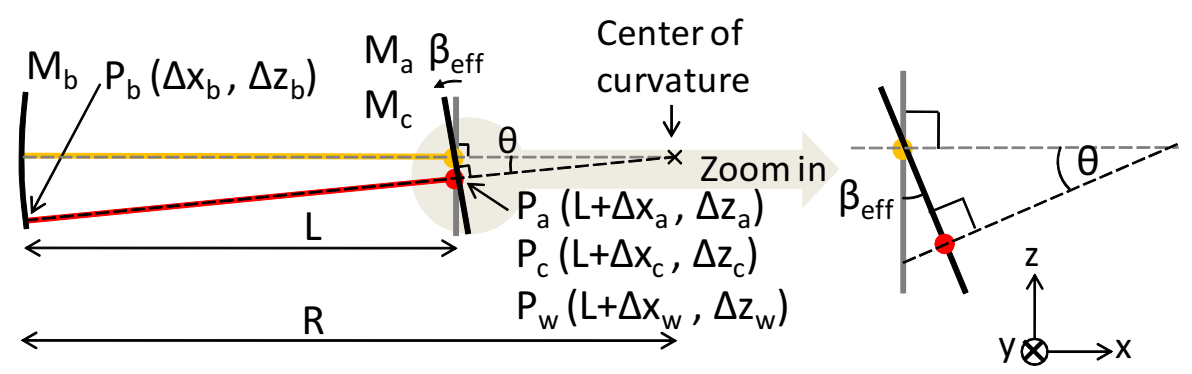

Figure 19. Projection of the triangular cavity onto the $x-z$ plane. It allows one to view the cavity as a plane cavity. The eigenmode is defined by the line that is orthogonal to the flat mirrors and passes through the center of curvature. In the right part, an enlarged cut-out around one flat mirror is shown.

that passes through the center of curvature and intersects the flat mirrors orthogonally, as described in [1]. The angle formed by the eigenmodes of the aligned and misaligned cases is denoted by $\theta$ in Fig. 19, and it becomes obvious that $\theta=\beta_{\text {eff }}$ when one focuses around the area of the flat mirrors, as shown in the enlarged cut-out in the right part of Fig 19. Therefore the following equations yield the spot position changes:

$$
\Delta x_{a, b, c, \text { and } w}=O\left(\beta_{+}^{2}\right)=0
$$


Eigenmode in a misaligned triangular optical cavity

$$
\begin{aligned}
& \Delta z_{a}=\Delta z_{c}=\Delta z_{w}=\beta_{\mathrm{eff}} \cdot(R-L)=\beta_{+} \cdot(R-L) / \sqrt{2} \\
& \Delta z_{b}=\beta_{\mathrm{eff}} \cdot R=\beta_{+} \cdot R / \sqrt{2}
\end{aligned}
$$

To summarize, a misalignment in $+(-) \beta_{+}$causes a counter-clockwise (clockwise) tilt of the geometrical axis of the two flat mirrors around the center of curvature. As a result the plane of the eigenmode tilts synchronously.

\subsection{Misalignment in $\beta_{-}$}

Here, mirrors $\mathrm{M}_{\mathrm{a}}$ and $\mathrm{M}_{\mathrm{c}}$ rotate around the $y_{a}$ and $y_{c}$-axis by $\pm 1 / 2 \beta_{-}$, respectively, as shown in Fig. 20. When the two opposite misalignment angles on mirrors $\mathrm{M}_{\mathrm{a}}$ and $\mathrm{M}_{\mathrm{c}}$ are projected onto

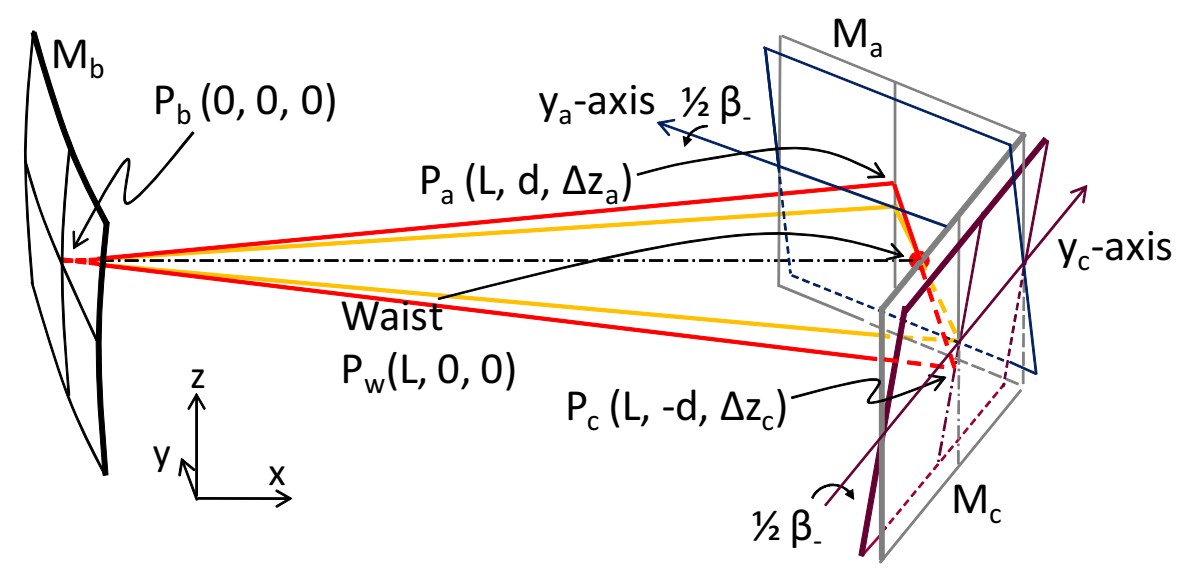

Figure 20. Cavity eigenmodes of the aligned and the misaligned $\left(\beta_{-}\right)$cases. The beam spot position and the waist position stays unchanged.

the $x-z$ plane, they appear as rotations around the $y$-axis by $\pm \frac{\beta_{\text {eff }}}{2}$, respectively, yielding no change along the $z$-axis on the curved mirror $\mathrm{M}_{\mathrm{b}}$. On the other hand, when they are projected onto the $y-z$ plane, as shown in Fig. 21, they both appear as rotations around the $z$-axis by $\beta_{\text {eff }} / 2$, yielding shifts along the $z$-axis in the beam spot positions on the two flat mirrors by the same amount, but with opposite sign. Note that here $\beta_{\text {eff }} \equiv \beta_{-} / \sqrt{2}$. These spot position changes are symmetrical along the $y$-axis, thus they do not yield a change in the beam spot position on the curved mirror along the $y$-axis, nor a change in the waist position (which is equidistant from the two spot positions) along the $y$-axis and $x$-axis. Hence the spot on the curved mirror and the waist remain unchanged, indicating that the new eigenmode is formed by rotating the aligned eigenmode around the $x$-axis by $\theta$, yielding no change in the lengths on any sides of the triangle.

The inclination angle of the beam between the two flat mirrors with respect to the $x-y$ plane is denoted by $\theta$ in Fig. 21. Focusing on the isosceles triangle as indicated by the shaded triangle in the figure, whose equal angles are denoted as $\mu$, the inclination angle is given by the following equations:

$$
\begin{aligned}
& \mu=\pi / 2-\beta_{\text {eff }} / 2 \\
& \theta=\pi-2 \mu=\beta_{\text {eff }}
\end{aligned}
$$

Therefore the beam spot position shifts on the two mirrors are calculated to be

$$
\Delta z_{a}=-\Delta z_{c}=d \cdot \alpha_{\mathrm{eff}}=d \cdot \beta_{-} / \sqrt{2}
$$




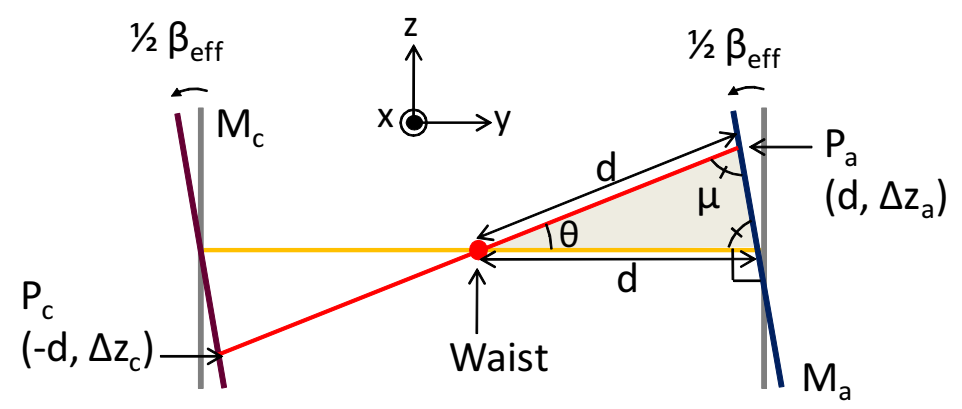

Figure 21. Projection of the triangular cavity onto the $y-z$ plane. The plane of the cavity is rotated around the $x$-axis by $\theta$, however the lengths of all the sides of the triangle remain unchanged.

To summarize, a misalignment in $+(-) \beta_{-}$causes no change in the spot position on the curved mirror and a counter-clockwise (clockwise) rotation of the non-congruent side around the $x$-axis. As a result, the plane of the eigenmode rotates synchronously.

\section{Result and comparison}

Tables 2 and 3 show the results from the geometrical analysis, and compare them to the simulation results obtained by using two simulation tools. One is OptoCAD [4] and the other is IFOCAD [5]. We used them to trace the Gaussian beam through our triangular cavity model that has the design parameters for the AEI $10 \mathrm{~m}$ Prototype reference cavity. These parameters are given as follows: $R=37.8 \mathrm{~m}, L=10.05 \mathrm{~m}$, and $d=0.15 \mathrm{~m}$. By inserting these values into our geometrical model, we obtained the corresponding numerical values. Due to the fact that OptoCAD is 2-dimensional we only used it for simulating the horizontal misalignment types.

\section{Conclusion}

The results of the geometrical analysis are in excellent agreement with the simulation results, showing sufficient accuracy for the design of an alignment control system for a triangular cavity. We have checked that all the discrepancies between the geometrical analysis and the simulations decrease by assigning real values for the two larger angles $\gamma$ to the geometrical analysis, instead of using $\gamma=\pi / 2$. This analysis can easily be extended to a cavity with more general shape if one follows the equations derived in this paper and modifies the method of approximation properly. The geometrical analysis not only serves as a method of checking a simulation result, but also gives an intuitive and handy tool to visualize the eigenmode of a misaligned triangular cavity.

\section{Acknowledgments}

This work was supported by the QUEST cluster of excellence of the Leibniz Universität Hannover. We like to thank Gerhard Heinzel for very helpful discussions on simulation challenges. 
Eigenmode in a misaligned triangular optical cavity

Table 2. Horizontal misalignment comparison.

\begin{tabular}{rrrrrrrrrrrr}
\hline Type & Method & \multicolumn{2}{c}{$\mathrm{M}_{\mathrm{a}}$} & \multicolumn{2}{c}{$\mathrm{M}_{\mathrm{c}}$} & \multicolumn{2}{c}{$\mathrm{M}_{\mathrm{b}}$} & \multicolumn{3}{c}{ Waist } \\
& & $\Delta x$ & $\Delta y$ & $\Delta x$ & $\Delta y$ & $\Delta x$ & $\Delta y$ & $\Delta x$ & $\Delta y$ & Angle \\
\hline \multirow{4}{*}{$\alpha_{b}$} & Geom.Analy. & 0.205 & -0.205 & -0.205 & -0.205 & 0 & -13.970 & 0 & 0.205 & -1.370 \\
& OptoCAD & 0.206 & -0.202 & -0.206 & -0.202 & 0 & -13.974 & 0 & 0.209 & -1.370 \\
& IfOCAD & 0.205 & -0.202 & -0.205 & -0.202 & 0 & -13.974 & 0 & 0.209 & -1.370 \\
\hline \multirow{4}{*}{$\alpha_{-}$} & Geom.Analy. & -10.051 & 10.051 & -10.051 & -10.051 & 0 & 0 & -10.051 & 0 & 0 \\
& OptoCAD & -10.051 & 9.902 & -10.051 & -9.902 & 0 & 0 & -10.051 & 0 & 0 \\
& IfOCAD & -10.051 & 9.903 & -10.051 & -9.903 & 0 & 0 & -9.9022 & 0 & 0 \\
\hline \multirow{4}{*}{$\alpha_{+}$} & Geom.Analy. & -0.151 & 0.151 & 0.151 & 0.151 & 0 & 0.205 & 0 & -0.001 & 1.005 \\
& OptoCAD & -0.151 & 0.149 & 0.151 & 0.149 & 0 & 0.206 & 0 & -0.003 & 1.005 \\
& IfOCAD & -0.151 & 0.149 & 0.151 & 0.149 & 0 & 0.205 & 0 & -0.003 & 1.005 \\
\hline
\end{tabular}

Table 3. Vertical misalignment comparison.

\begin{tabular}{rrrrrrrrrrr}
\hline Type & Method & \multicolumn{2}{c}{$\mathrm{M}_{\mathrm{a}}$} & \multicolumn{2}{c}{$\mathrm{M}_{\mathrm{c}}$} & \multicolumn{2}{c}{$\mathrm{M}_{\mathrm{b}}$} & & Waist \\
& & $\Delta x$ & $\Delta z$ & $\Delta x$ & $\Delta z$ & $\Delta x$ & $\Delta z$ & $\Delta x$ & $\Delta z$ & Angle \\
\hline$\beta_{b}$ & Geom.Analy. & 0 & 37.800 & 0 & 37.800 & 0 & 37.800 & 0 & 37.800 & 0 \\
& IfocAD & 0 & 37.800 & 0 & 37.800 & 0 & 37.800 & 0 & 37.800 & 0 \\
\hline$\beta_{-}$ & Geom.Analy. & 0 & 0.106 & 0 & -0.106 & 0 & 0 & 0 & 0 & 0.707 \\
& IfocAD & 0 & 0.106 & 0 & -0.106 & 0 & 0 & 0 & 0 & 0.702 \\
\hline$\beta_{+}$ & Geom.Analy. & 0 & -19.622 & 0 & -19.622 & 0 & -26.729 & 0 & -19.622 & 0 \\
& IfOcAD & 0 & -19.770 & 0 & -19.770 & 0 & -26.930 & 0 & -19.770 & 0 \\
\hline
\end{tabular}

\section{References}

[1] Heinzel G 1999 Advanced optical techniques for laser-interferometric gravitational-wave detectors, PhD thesis ftp.rzg.mpg.de/pub/grav/ghh/ghhthesis.pdf

[2] Kawazoe F et al. 2010 Designs of the frequency reference cavity for the AEI $10 \mathrm{~m}$ prototype interferometer, J. Phys. Conf. Ser. textbf228 012028

[3] Freise A 2010 Frequency domain INterfErometer Simulation SotfwarE, FINESSE manual http://www.gwoptics.org/finesse/download/Finesse-0.99.8.pdf

[4] OptoCAD (0.90c) 2010 A Fortran 95 module for tracing Gaussian TEM $_{00}$ beams through an optical set-up, written by Roland Schilling

Simulation tool http://www.rzg.mpg.de/ ºs/optocad.html

[5] IfoCAD 2010 A framework of C subroutines to plan and optimize the geometry of laser interferometers, written by Gerhard Heinzel

Simulation tool 